COMENTARIO

\title{
Una experiencia de gestión participativa de la biodiversidad con comunidades amazónicas
}

\section{An experience on participatory management of biodiversity with Amazonian communities}

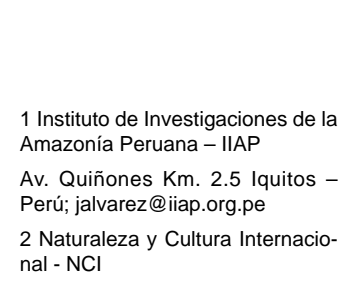

nal $-\mathrm{NCl}$ $\begin{array}{ll}\text { Presentado: } & 24 / 01 / 2012 \\ \text { Aceptado: } & 12 / 09 / 2012\end{array}$ Publicado online: $10 / 11 / 2012$

\author{
José Álvarez ${ }^{1}$ y Noam Shany ${ }^{2}$
}

\section{Resumen}

El Instituto de Investigaciones de la Amazonía Peruana - IIAP, Nature and Culture International (NCI) junto con comunidades locales, el Gobierno Regional de Loreto y otras organizaciones privadas, han estado desarrollando en la última década un modelo de cogestión comunitaria de la biodiversidad amazónica, con resultados muy promisorios. En el presente trabajo se analizan los principales logros de este modelo. En las tres cuencas donde se ejecutaron estos proyectos (Tahuayo, Yanayacu del Amazonas y Nanay) observamos la reversión de los procesos de degradación de recursos y eco sistemas, la reducción significativa de la tala, pesca y caza ilegales, y de la cosecha destructiva de recursos; también han mejorado significativamente algunos indicadores económicos de las poblaciones locales involucradas gracias a la comercialización de productos con valor agregado y la recuperación de las pesquerías, la fauna silvestre y otros recursos. Las líneas maestras de este enfoque fueron: (1) conservación productiva (conservación como negocio, esto es, recuperar recursos de flora y fauna silvestres y conservarlos productivamente para la gente, generando ingresos); (2) manejo adaptativo (medidas sencillas de manejo diseñadas y adoptadas progresivamente por las propias comunidades con apoyo de los técnicos, siguiendo el modelo de investigación participativa); (3) enfoque ecosistémico (conservación de grandes paisajes, especialmente cuencas y ecosistemas completos, incluyendo procesos ecológicos priorizados, como migraciones estacionales de peces, y conservación de áreas fuente); (4) uso y rentabilización del bosque en pie (priorizar generación de ingresos de recursos silvestres manejados vs. cambio de uso del bosque); (5) participación de todos los actores, desde las comunidades locales organizadas debidamente consultadas hasta las empresas privadas, las instituciones públicas y los tomadores de decisión; (6) desarrollo de cadenas productivas completas, con agregación de valor a productos de la biodiversidad y articulación al mercado; (7) gestión adaptativa (decisiones tomadas localmente, y adecuación progresiva de las instituciones y normas de acuerdo a las capacidades de las comunidades).

Palabras clave: Amazonia peruana; conservación productiva; manejo; cogestión; comunidades indígenas.

\section{Abstract}

For the past 10 years, the Instituto de Investigaciones de la Amazonía Peruana (Peruvian Amazon Research Institute) - IIAP, Nature and Culture International $(\mathrm{NCl})$, together with local communities, the Regional Government of Loreto, and other private organizations, have been developing a community co-management model for the Amazonian biodiversity, with very promising results. In the three basins where such co-management projects were implemented (Tahuayo, Yanayacu and Nanay), we are seeing a concrete evidence of reversals in the processes of degradation of resources and ecosystems. In each of these basins, a significant reduction in logging, illegal fishing and hunting, and destructive harvesting methods of resources can be found as a directresult of the co-management model. Significant economic improvements to local communities are achieved mainly due to the commercialization of value-added biodiversity products, as well as the recovery of fisheries, wildlife and other resources. The main principles of this model are : (1) productive conservation (conservation as a business, that is to recover flora and fauna resources and their management in ways that allow income generation); (2) adaptive management (simple management actions designed and progressively adopted by the communities and accompanied with technical support); (3) eco-systemic approach (conservation of large landscapes, especially watersheds, prioritized ecological processes such as fish's seasonal migrations, and conservation of source areas); (4) making use of standing forest (to prioritize revenues from sustainable use of forest vs. cutting down the forest); (5) participation of all stakeholders from the organized and properly consulted local communities, to private enterprises, public institutions and all decision-makers; (6) development of complete productive chains with value-added biodiversity products and insertion in markets; (7) adaptive management (progressive adjustment of institutions and norms according to communities' capacities).

Keywords: Peruvian Amazonia; productive conservation; management; co-management; indigenous communities.

\section{Introducción}

Los bosques amazónicos, los más extensos del Mundo en los trópicos, son considerados un elemento clave en el equilibrio climático global, además de una reserva estratégica de recursos genéticos y de agua dulce. Estos bosques están mucho más amenazados de lo que se pensaba, y no solamente por la deforestación para fines agrícolas, sino por la degradación de hábitat y por el cambio climático. Nuevas tecnologías de detección remota han demostrado que la degradación del bosque amazónico provocada por la tala selectiva, en su mayor parte ilegal, es mucho más grave de lo calculado anteriormente, y contribuye en aproximadamente un $27 \%$ a las emisiones de carbono atribuidas a la deforestación (Asner et al. 2010). Por otro lado, se ha comprobado que los bosques amazónicos no son tan resilientes como se había pensado, y son muy susceptibles a las sequías que han asolado la Amazonía en los últimos años (Phillips et al. 2009). La degradación de los ecosistemas amazónicos está provocando, a su vez, el deterioro de la calidad de vida de las poblaciones amazónicas que dependen para su subsistencia de los recursos de flora y fauna. 
Las nuevas amenazas que han surgido para los bosques amazónicos están relacionadas con la tala selectiva de madera, la minería aluvial y la ampliación de la frontera agrícola, incluyendo el incremento de los cultivos de estupefacientes y, en los últimos años, con los agrocombustibles. Estos últimos, y especialmente la palma aceitera o palma africana (Elaeis guineensis) son vistos por algunos como la mayor amenaza para los bosques amazónicos en las próximas décadas (junto con la soya), aunque han sido impulsados paradójicamente para ayudar a combatir el cambio climático (Butler \& Laurance 2009; Houtart 2011). Especialmente preocupante es la expansión de los cultivos de palma aceitera en la Amazonía peruana: pese a que sus impulsores plantean el uso de áreas deforestadas, en la mayoría de los casos las plantaciones se establecen a costa de bosques primarios, con frecuencia sobre territorios de uso tradicional por comunidades locales (Smolker et al. 2007).

Pero el bosque amazónico sufre otras amenazas no tan visibles. La defaunación debida a la sobre caza de animales silvestres es tan grave que puede estar poniendo en riesgo la funcionalidad de los ecosistemas y la misma composición de la vegetación (Terborgh 1999; Peres 2000; Terborgh et al. 2008). Algunos científicos hablan del síndrome del "bosque vacío", en alusión a la escasez creciente de grandes animales, muchos de los cuales cumplen un importante rol en el ecosistema como dispersores de semillas o controladores de vegetación (Redford 1992). La escasez de animales silvestres también tiene consecuencias sociales, pues se está incrementando la desnutrición en las poblaciones locales que dependen de este recurso como fuente de proteína (Álvarez 2007).

Por otro lado, la creciente preocupación por el cambio climático y la crisis económica global ha contribuido a una aparente disminución de la preocupación por la conservación de la biodiversidad, a la par que ha disminuido el financiamiento para proyectos orientados a esta línea. También han surgido crecientes críticas contra un conservacionismo poco preocupado por los temas de desarrollo y pobreza. Con frecuencia se ha impulsado la conservación sin tener en cuenta a los actores locales, con nefastas consecuencias: 'parques de papel' caros de mantener e insostenibles en el tiempo, dinero malgastado en fracasados proyectos conservacionistas no sostenibles. (Brown 2003; Ferraro \& Pattanayak 2006)

El aprovechamiento integral del bosque en pie (incluyendo la producción forestal sostenible - PFS, de productos forestales maderables y no maderables, y de servicios ambientales, SA) es visto como una de las alternativas para enfrentar la conversión de bosques en áreas de cultivo y pastoreo, y la degradación por tala selectiva y caza descontroladas. En términos de rentabilidad, la combinación de estos dos usos del bosque (PFS y SA, especialmente el mercado de carbono) es la única que puede competir en rentabilidad con la conversión de bosques a cultivos de palma aceitera (Butler \& Laurance 2009).

La imparable ola de destrucción de los bosques tropicales ha dado origen a un interesante debate en las últimas décadas sobre el rol en el manejo de los recursos naturales de las comunidades locales, especialmente las indígenas, que viven en esos bosques, debate que ha sido llevado a las mesas de negociación sobre el cambio climático; una menor atención ha sido puesta en el rol de las numerosas comunidades ribereñas o mestizas que viven en las riberas de los ríos amazónicos más grandes, y que por estar más vinculados al mercado tienen patrones de uso del territorio algo diferentes, con más énfasis en las actividades agropecuarias (Anderson 1989; Goodman \& Hall 1990; Davis \& Wali 1994).

Se ha puesto mucho énfasis en fortalecer los derechos de los indígenas sobre el territorio, dando por hecho que la conservación de los bosques se va a dar una vez que estos tengan el control total; muchos autores que han estudiado las diversas culturas indígenas resaltan el profundo respeto que las sociedades indígenas tradicionales tienen (o más bien, tenían) por la naturaleza y la necesidad de preservar esas cosmovisiones y prácticas tradicionales para garantizar la conservación de los bosques, ignorando en cierto modo los incontenibles y cada vez más acelerados procesos de aculturación e inserción en la economía de mercado (Redford \& Stearman 1993). Sin embargo, la gran mayoría de los estudios sobre comunidades indígenas ha sido hecha en comunidades muy tradicionales, y se ha dejado de lado las comunidades más vinculadas al mercado y, por tanto, más aculturadas y 'menos conservacionistas", aun cuando el proceso de tránsito de unas a otras es cada vez más acelerado, y aparentemente irreversible e inevitable (Johnson 1989; Holt 2005; De Soto 2010). La experiencia demuestra que también los indígenas pueden ser partícipes de destrucción de los bosques tropicales, o al menos ser testigos pasivos, cuando son dejados a merced de las fuerzas de la globalización sin instrumentos técnicos y legales para gestionar sus bosques y beneficiarse económicamente de ellos (Redford \& Taber 2000).

Algunos autores ya hace tiempo hicieron notar que los indígenas pueden ser aliados de la conservación siempre y cuando, además de control efectivo y la seguridad legal de sus territorios, dispongan de capacitación y herramientas técnicas para el manejo sostenible y rentable de sus recursos (Clad 1984; Davis \& Wali 1994; Peres 1994). Un reciente estudio demuestra que los bosques en manos de comunidades locales (en 16 países de América Latina, África y Asia) sufren tasas de deforestación significativamente menores que aquellos dentro de áreas naturales protegidas de forma estricta: las áreas protegidas perdieron en promedio $1,47 \%$ de su cobertura boscosa al año, en comparación con $0,24 \%$ de los bosques manejados por comunidades (Porter-Bolland et al. 2011).

Debemos partir del hecho de que las culturas y sociedades indígenas y mestizas amazónicas no son estáticas, como no lo son sus usos de los recursos silvestres y sus formas de organización. La variabilidad ecológica y la dinámica histórica han impulsado a las sociedades indígenas a adaptarse y a crear constantemente formas nuevas de uso del territorio y los recursos (Clay 1988; Posey \& Balee 1989). Sus sistemas de creencias, que regulaban en el pasado el uso del territorio y los recursos, a través de tabúes y otros mecanismos, se enfrentan con desventaja al avasallador avance de la cultura y economía occidentales, y terminan colapsando si las comunidades indígenas no encuentran alternativas económicas social, ambiental y económicamente sostenibles. Muchos proyectos de conservación en la Amazonía han fracasado justamente en el mediano plazo, o bien por desconocer las particularidades socioculturales de las comunidades involucradas, o por descuidar el aspecto de sostenibilidad económica, generalmente en temas de valor agregado y mercado (Gasché 2004; Álvarez 2007).

La creciente emigración de las zonas rurales a la ciudades grandes y medianas ha sido vista como una esperanza para la conservación de los bosques tropicales (Wright \& Muller-Landau 
2006); sin embargo, el acelerado crecimiento de las ciudades amazónicas está generando grandes impactos en las áreas más cercanas, y no está mitigando la sobre explotación de los recursos de flora y fauna en las zonas alejadas, adonde extractores no residentes siguen con sus actividades para abastecer la demanda de las ciudades, generalmente sin manejo y con prácticas destructivas de cosecha (Parry et al. 2010).

\section{Comunidades locales, aliadas de la conservación}

Ante las altas tasas de deforestación y la imbatible disminución y fragmentación de los bosques tropicales prístinos, algunos dan por hecho que no podrán ser conservadas grandes extensiones de bosques primarios, y ponen énfasis en la conservación de los agroecosistemas, especialmente los mosaicos de hábitat relacionados con la agricultura tradicional (Vandermeer \& Perfecto 2007b). Quienes se han resignado a perder las últimas grandes extensiones de los bosques tropicales están abogando por nuevas e innovadoras formas de conservar en un escenario dominado por comunidades y pequeńos agricultores con títulos de propiedad sobre bosques y áreas agrícolas: plantean conservar no solo fragmentos de los bosques remanentes (incluyendo las áreas protegidas), sino la matriz que incluye áreas manejadas de bosques y áreas de cultivo tradicionales favorables para la biodiversidad (sistemas agroforestales y otros; Roe \& Jack 2001; Schwartzman \& Zimmerman 2005; Vandermeer \& Perfecto 2007a). Se impone la idea de que las alianzas y colaboración con las comunidades locales y con los movimientos y organizaciones de campesinos e indígenas será crucial para la conservación de la biodiversidad en general y de los bosques tropicales en particular (Vandermeer \& Perfecto 2007b). Aunque los objetivos de estos movimientos suelen estar vinculados principalmente a la defensa de sus derechos sobre la tierra y otros recursos (agua, bosques, etc.), el tema ecológico está cada vez más presente, y en sus mensajes y acciones aparece la conservación de los ecosistemas y la biodiversidad como un objetivo estrechamente ligado con la defensa de su modo de vida (ver por ej. Wright $\&$ Wolford 2003).

Cabe destacar que en la Región Loreto el escenario es favorable todavía para la conservación y manejo sostenible de grandes extensiones de bosques primarios: apenas un 3\% de las 36.8 millones de hectáreas de bosques han sido transformados por la agricultura.

Por otro lado, existe una visión bastante pesimista de que la conservación de los bosques tropicales no podrá sostenerse en argumentos económicos ("el bosque amazónico vale más vivo que muerto") y las opciones de desarrollo siempre son priorizadas frente a las de conservación, frente a lo que se plantea el fortalecimiento de los argumentos éticos (Terborgh 1999; Sanderson \& Redford 2003)

En un escenario en que disminuyen los fondos para conservación a la par que se incrementan las amenazas contra los bosques amazónicos, las comunidades locales son vistas cada vez más no tanto como una amenaza sino como potenciales aliados de la conservación, como parte de la solución en vez de parte del problema (Vandermeer \& Perfecto 2007a; Vermeulen \& Sheil 2007). En los últimos años se ha puesto de moda en enfoque de la "participación" en los proyectos y actividades de conservación en países tropicales; la mayoría de ellos, sin embargo, y a pesar de la retórica "participativa", siguen siendo diseñados y controlados por organizaciones y pequeños grupos de expertos; en ellos siguen estando ausentes tanto la consulta formal como las formas democráticas en la ejecución de los proyectos, al tiempo que se ignoran en buena medida las iniciativas, prioridades e intereses de las comunidades locales (Campbell \& Vainio-Mattilia 2003; Vermeulen \& Sheil 2007).

La participación se ha convertido en algunos casos en una especie de ritual que todo proyecto incluye sin mayor criterio, y puede convertirse en un elemento distorsionador: la participación de las comunidades tanto en el cuidado de los recursos como en el monitoreo no es sostenible si los pobladores locales no ven retribuido su esfuerzo con beneficios claros y tangibles (Cooke $\&$ Kothari 2001). Si bien muchos conservacionistas están de acuerdo en la necesidad de establecer alianzas con las comunidades locales en la búsqueda de objetivos comunes y líneas coincidentes para conservar la biodiversidad y los ecosistemas tropicales, hay mucha polémica sobre el cómo. Los 'joint ventures' y otros negocios compartidos con comunidades han sido vistos como una de las mayores oportunidades para la conservación y el desarrollo sostenible en bosques tropicales (CSD 2004).

Un intenso debate también ha surgido en torno a la necesidad de establecer alianzas (partnerships) entre comunidades indígenas, científicos y profesionales de la conservación, gobiernos y agencias de cooperación internacional (Vermeulen \& Sheil 2007). Los bionegocios con base en los incalculables recursos genéticos amazónicos y el incipiente mercado de carbono se avizoran como muy positivas oportunidades para impulsar productivas y estratégicas alianzas entre las comunidades locales, empresas, gobierno y organizaciones conservacionistas y de cooperación, en la búsqueda de un desarrollo realmente inclusivo y sostenible. Sin embargo, estas alianzas deben que tener objetivos claros compartidos, altos estándares de participación -incluyendo decisiones, derechos y deberes, y riesgos compartidos- y deben beneficiar claramente a todas las partes, y especialmente a las comunidades locales involucradas (Campbell \& Vainio-Mattilia 2003; Vermeulen \& Sheil 2007).

Existen diversas y promisorias experiencias de colaboración entre científicos conservacionistas y comunidades, por ejemplo en el tema de manejo de fauna silvestre y en monitoreo de la biodiversidad (Bodmer et al. 1997). Pese a que algunos científicos ponen en cuestión su "precisión científica", el monitoreo comunitario o participativo con comunidades ha demostrado ser sumamente efectivo en términos de costo-resultados y de impacto en la gestión, que en definitiva es lo que más importa. Además, las comunidades involucradas en monitoreo suelen mejorar el control sobre sus territorios y recursos, lo que contribuye a la conservación de la biodiversidad (Getz et al. 1999; Sheil \& Lawrence 2004; Danielsen et al. 2007).

Las estrategias de conservación de los ecosistemas a través de la creación de áreas protegidas (especialmente parques nacionales) y compra de tierras están llegando a su techo y se manifiestan insostenibles, debido a los excesivos costos sociales y económicos (Ghimire \& Pimbert 1997; Bruner et al., 2001; Balmford \& Whitten 2003). Muchas de las iniciativas de conservación se ven frenadas por la oposición de comunidades locales y organizaciones que las apoyan, porque no han sido consultadas de forma adecuada, y porque se sienten limitadas en sus aspiraciones de acceso a los recursos silvestres; también se oponen sectores económicos interesados en el aprovechamiento de recursos, que ven en las áreas protegidas una amenaza a sus intereses. 
En la Amazonía peruana en particular, la cobertura de las formas tradicionales de áreas protegidas está llegando a su límite (aproximadamente un 20\% del territorio, y en algunas regiones, como Madre de Dios, aún más) y debe buscarse alternativas para proteger grandes extensiones de bosques todavía en el limbo legal de "áreas de libre disponibilidad del Estado", donde persiste buena parte de la biodiversidad. Además, cada vez es más evidente que la conservación de la biodiversidad amazónica y los servicios y recursos que proveen los bosques amazónicos requieren del mantenimiento de grandes y continuas extensiones de bosques y la protección de los procesos ecológicos y evolutivos que hacen posible la biodiversidad (García Villacorta 2010).

En la Región Loreto, en la Amazonía norperuana, tenemos una oportunidad histórica para conservar grandes extensiones de bosques tropicales porque aquí todavía los bosques valen más vivos que muertos y se conserva todavía el $97 \%$ de los bosques originales, 36.8 millones de hectáreas en total. La frontera agrícola es incipiente, y gran parte de las cerca de 3,000 comunidades locales, tanto indígenas como ribereñas, basan su economía en el aprovechamiento sostenible de los recursos forestales y de fauna silvestre (Pyhälä 2003; Álvarez 2006).

En este documento se describen algunas experiencias que demuestran que el bosque amazónico puede valer más vivo que muerto, en pie que talado, contradiciendo la pesimista visión de algunos expertos, incluyendo la de J. Terborgh (1999). Innovadoras formas de concebir las áreas protegidas como zonas de aprovechamiento sostenible de recursos por comunidades locales, manteniendo la integridad y salud de los ecosistemas, han cambiado en cierto modo el escenario, abriendo la posibilidad de crear nuevas áreas en alianza con comunidades locales interesadas en conservar las zonas fuente de sus recursos de fauna y flora (Bodmer 2000; Bodmer \& Puertas 2000).

\section{Una visión tergiversada de la Amazonía}

En las últimas décadas se han invertido decenas de millones de dólares de la cooperación internacional y del presupuesto público del Perú para impulsar proyectos de desarrollo y conservación con comunidades locales amazónicas, con resultados irrisorios, o en muchos casos contraproducentes. Las amenazas contra biodiversidad crecen día a día, a la par que se deteriora la calidad de vida de las poblaciones amazónicas que dependen de los recursos de flora y fauna para su subsistencia. Los índices de pobreza y desnutrición en la selva son los peores del Perú, y en algunos casos han empeorado, a contracorriente de lo que ocurre en la mayor parte del país, donde ha ocurrido una significativa reducción de la pobreza.

El fracaso de la mayoría de los proyectos (tanto de conservación como de desarrollo) se debe -a nuestro juicio y de algunos autores- a la falta de comprensión tanto de la realidad ecológica de la Amazonía baja como de las sociedades amazónicas que viven en ella (Gasché 2004). Parte del problema reside en el divorcio entre quienes impulsan la conservación de la biodiversidad y los que impulsan el desarrollo de las comunidades (Guha 1997; Holt 2005). Por ejemplo, la mayoría de los proyectos de desarrollo con comunidades impulsaron actividades agropecuarias copiadas de otras realidades, no apropiadas para los pobres y complejos suelos amazónicos, ni acordes con la tradición extractivista y las formas de organización de los pobladores locales (CADMA 1992; Álvarez 2007).
Para citar un ejemplo: desde un tiempo a esta parte se ha dado en promover en Amazonía proyectos productivos "comunales", cuando la 'comunidad' no es una unidad social de producción, y el modo de producción tradicional es familiar, o de familias extensas (Gasché 1999; 2010). De este modo han fracasado cientos de proyectos de piscigranjas familiares, crianzas comunales de animales domésticos, chacras comunales, etc.

La Amazonía peruana ha sido históricamente abordada desde el Gobierno Nacional asentado en la costa (Lima, la capital) con una visión sesgada de su realidad ecológica y social. No solo se trata de los mitos sobre la Amazonía que aún sobreviven en el imaginario colectivo nacional (mitos de la uniformidad, del vacío amazónico, de la fertilidad de los suelos - CADMA 1992) sino de una visión del desarrollo calcada de otras realidades, ajenas a los ecosistemas y las sociedades amazónicas. Pero la Amazonía es diferente: primero, por su extraordinaria riqueza natural y cultural, que se expresa en su extremada diversidad de ecosistemas, especies genes y culturas humanas; y segundo, por su extremada fragilidad. El manejo de la biodiversidad en Amazonía enfrenta retos y problemas diferentes a los de otras latitudes. Por ejemplo, son escasas y poco efectivas las medidas y técnicas de manejo aplicables en los complejos ecosistemas amazónicos (y aplicadas con éxito en países templados), debido especialmente al régimen de libre acceso a los recursos, a la inaccesibilidad de muchas áreas de extracción, a la enorme variabilidad ecológica de los ecosistemas amazónicos, al escaso conocimiento disponible sobre historia natural, densidad y dinámica poblacional de la mayoría de las especies, a la idiosincrasia de las poblaciones locales, y a las limitaciones de las instituciones responsables de hacer cumplir la ley.

Los funcionarios públicos y miembros de organizaciones de cooperación que se han acercado a la Amazonía tratando de impulsar proyectos de desarrollo la han visto generalmente como un suelo a cultivar, y no han podido ver el bosque (o han visto solamente madera comercial en él), ni las formas tradicionales de aprovecharlo que tienen las comunidades locales. Desde tiempos del Presidente Belaúnde Terry (en sus dos gobiernos, a fines de los 60 y principios de los 80 ), con su lema "Tierra sin gente para gente sin tierra”, y su visión de la Amazonía como "arroz con bisteck", se ha impulsado proyectos agropecuarios calcados de la sierra o la costa del Perú, absolutamente inapropiados para los suelos pobres y ácidos de la selva baja, y no acordes con la cultura y formas de producción de las comunidades amazónicas tradicionales (CIAM 2011).

No es por eso extraño que la Amazonía peruana haya sido acertadamente calificada como un "cementerio de proyectos". No hay comunidad indígena o ribereńa que no tenga en su historia algún proyecto de desarrollo fracasado, sea impulsado por alguna agencia del Gobierno o por alguna organización no gubernamental. La mayoría de estos proyectos han estado y están relacionados con la producción agrícola o pecuaria, incluyendo promoción de cultivos comerciales, reforestación con especies nativas o introducidas, crianza de animales (menores o mayores), tanto nativos como introducidos, piscicultura familiar o comunal, y otros similares. En otros casos son las obras de infraestructura, con bastante frecuencia sin un sentido productivo ni el mínimo sentido de la prioridad: plazas, veredas, losas deportivas, puentes sobre minúsculas quebradas...

El enfoque excesivamente técnico de la mayoría de los proyectos, ajeno en buena medida a las aspiraciones, capacidades 
y visión de la población local, ha sido identificado como otra de las causas más comunes de fracasos de proyectos, los que suelen ser dirigidos por profesionales muy especializados con una visión muy sesgada de la realidad (Gasché 2004; Santoyo 2004). Sin embargo, también ciertas visiones antropológicas, con su excesivo -y con frecuencia exclusivo- énfasis en aspectos socioculturales, y con limitado conocimiento e interés en temas ecológicos y productivos, han ayudado poco a promover modelos de desarrollo sostenible en las comunidades amazónicas.

Por otro lado, la mayoría de los proyectos de desarrollo y conservación han sido concebidos con un muy limitado horizonte espacial y temporal: se trata de intervenciones en una sola o un puñado de comunidades, con frecuencia no relacionadas espacialmente, y de corto plazo; en los aspectos productivos, la mayoría no consideran la cadena productiva completa -comenzando del aprovechamiento sostenible del recurso-, y menos aún aspectos de gestión empresarial y de mercado. Esto determina casi de antemano su insostenibilidad. Para garantizar la sostenibilidad ecológica es necesario gestionar con enfoque ecosistémico grandes espacios, preferiblemente cuencas completas, y para garantizar sostenibilidad económica debe promoverse la agregación de valor, el fortalecimiento de capacidades empresariales y la articulación con los mercados.

Por poner algunos ejemplos del tema ecológico: no tiene mucho sentido, ni impacto ciertamente, tratar de manejar solamente un pequeño lago amazónico, y menos una sola especie comercial del lago, cuando la mayoría de los peces son grandes emigrantes, y también necesitan el bosque inundable circundante y de otros ecosistemas fuera del lago para alimentarse y reproducirse. Muchas especies de fauna requieren de grandes territorios para mantener poblaciones saludables y viables: no es posible manejar, por ejemplo, ungulados o grandes primates en el territorio de una sola comunidad, por muy grande que sea, porque no alberga poblaciones viables; es indispensable, por eso, trabajar con una visión de paisaje, integrando grandes espacios y trabajando con todas o las mayoría de las comunidades y sus organizaciones representativas de una cuenca, así como con otros actores que intervienen en una cuenca o zona determinada. Hoy se sabe que para que la caza de animales silvestres amazónicos sea sostenible es necesario proteger 'zonas fuente' conectadas con las zonas de caza, donde los animales se puedan reproducir y dispersarse hacia las zonas de caza (Bodmer 2000; Ferraro \& Pattanayak 2006)

La falta de sostenibilidad ambiental tiene otras caras: la mayoría de los proyectos de desarrollo en Amazonía peruana centran sus esfuerzos en promover alternativas productivas con base en especies cultivadas o criadas en cautividad (cultivos nativos o, con frecuencia, exóticos, y crianza de animales exóticos, incluyendo ganadería extensiva), promoviendo con ello directa o indirectamente la desvalorización del bosque y la deforestación. Son muy escasos los proyectos e iniciativas, tanto de las instituciones del Estado como de la cooperación internacional, que busquen generar recursos económicos aprovechando el bosque en pie (esto es, manejo de recursos silvestres de flora y fauna). Sin embargo, la mayor parte de los pobladores rurales amazónicos siguen usando en gran medida los recursos silvestres, forestales y acuáticos, para fines de subsistencia y también para obtener recursos económicos con su venta; por ejemplo, el pescado y la carne de animales silvestres representan entre el 70 y el $80 \%$ de la ingesta de proteína en buena parte de las familias rurales en Loreto. Un estudio realizado en comunidades del bajo Nanay, bien conectadas con el mercado de Iquitos, mostró que alrededor del $70 \%$ de los ingresos familiares provienen de la venta de recursos silvestres, especialmente recursos forestales maderables y no maderables, carne de monte, frutos silvestres y pescado (Pyhälä 2003). Solamente en las comunidades muy cercanas a las ciudades y pueblos grandes, y en las riberas de los grandes ríos de agua blanca (con suelos aluviales relativamente fértiles) la agricultura representa un porcentaje importante de los ingresos.

\section{Trabajando con comunidades locales y conser- vando los bosques}

Desde finales de la década de 1990, el Instituto de Investigaciones de la Amazonía Peruana - IIAP, consciente de esta situación y ante la imparable degradación de los recursos naturales renovables y de la calidad de vida de las poblaciones amazónicas, se propuso impulsar proyectos que buscasen combinar la conservación de la diversidad biológica y de los ecosistemas y el desarrollo sostenible de la población, con una sólida base ecológica y socioeconómica, con visión ecosistémica y de grandes paisajes, y con participación activa de las comunidades locales involucradas.

El primer proyecto impulsado por el IIAP se ejecutó entre el ańo 2000 y el 2004 con apoyo del Banco Mundial (y con fondos del Global Environmental Fund) en la cuenca del río Nanay, Loreto, Perú. El IIAP se planteó el reto de experimentar un modelo de desarrollo sostenible y conservación de la diversidad biológica en una cuenca emblemática de la Amazonía peruana; se escogió el Nanay por ser ejemplo de la extremada riqueza biológica y variabilidad de ecosistemas de la región, y por presentar un panorama extremo de depredación y degradación de los ecosistemas, dada su cercanía y conectividad con Iquitos. Se sabía de muchos proyectos fallidos de desarrollo en esta cuenca.

La experiencia del Proyecto "Conservación de la biodiversidad y manejo comunal de los recursos naturales en la cuenca del río Nanay" (Proyecto Nanay) demuestra que sí es posible aplicar con éxito medidas de conservación de la diversidad biológica y de desarrollo sostenible con comunidades locales, con las siguientes estrategias de intervención: 1) Enfoque integral y de cuenca: manejo integral del ecosistema, preferible al nivel de cuenca; 2) enfoque de "conservación productiva": conservar para la gente, generar riqueza sin alterar los ecosistemas; la población conserva lo que le es útil; 3) definición clara de derechos de acceso a los recursos $y$, especialmente, control del territorio por parte de comunidades; 4) diagnóstico participativo de la problemática de los recursos naturales; 5) apoyo a la organización interna de las comunidades, capacitadas y fortalecidas para el manejo (asambleas y grupos comunales de manejo), y a la organización supracomunitaria (comités zonales de gestión, federaciones); 6) diseño participativo y aplicación de planes de manejo adaptativo al nivel de comunidad; 7) co-gestión, aplicación de reglamentos comunales internos de acceso a los recursos con apoyo de los técnicos y las instituciones del Estado; 8) formalización / legalización del aprovechamiento de los recursos; 9) alianzas estratégicas con instituciones públicas y organizaciones de cooperación; y 10) diversificación de la base productiva y acceso al mercado (IIAP 2004; Álvarez 2006).

En la Tabla 1 se muestra un ejemplo de los avances conseguidos en la disminución de amenazas en 16 comunidades de 
Tabla 1. Disminución de las amenazas para los recursos naturales en 16 comunidades de la cuenca del Nanay.

\begin{tabular}{|c|c|c|c|c|c|}
\hline \multirow[t]{2}{*}{ Recurso/actividad } & \multirow[b]{2}{*}{ Año } & \multicolumn{3}{|c|}{$\mathbf{N}^{\circ}$ de extractores ilegales de: } & \multirow[b]{2}{*}{ Disminución (\%) } \\
\hline & & 2002 & 2003 & 2004 & \\
\hline Irapay & & 164 & 133 & 24 & 85 \\
\hline Madera redonda & & 201 & 249 & 44 & 82 \\
\hline Madera aserrío & & 185 & 162 & 20 & 89 \\
\hline Pesca & & 514 & 841 & 224 & 73 \\
\hline \multirow[t]{3}{*}{ Árboles maderables para leña } & & 72 & 63 & 16 & 77 \\
\hline & & \multicolumn{3}{|c|}{ Cosecha destructiva de RR.NN. (\%) } & \\
\hline & Año & 2002 & 2003 & 2004 & \\
\hline Ungurahui & & 100 & 50 & 10 & 90 \\
\hline Aguaje & & 100 & 49 & 9 & 91 \\
\hline Irapay & & 74 & 31 & 7 & 51,3 \\
\hline Madera redonda & & 54 & 32 & 6.3 & 70,3 \\
\hline Pesca destructiva & & 29 & 16 & 0 & 55,1 \\
\hline Tala ilegal & & 36 & 21 & 3 & 66,6 \\
\hline
\end{tabular}

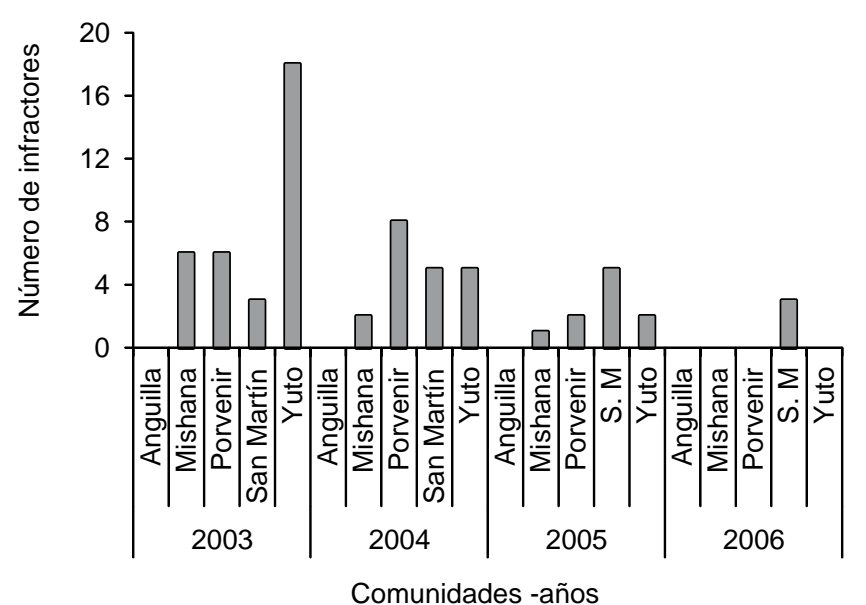

Figura 1. Índice de extractores ilegales de irapay en comunidades de la Reserva Nacional Allpahuayo-Mishana (RNAM) (Fuente: Informes finales del Proyecto BIODAMAZ Fase II. IIAP, Iquitos, 2007. http:// www.iiap.org.pe/biodamaz/faseii/avances/Doc_tec.htm)

la cuenca, en este caso la comunidad nativa San Juan de Ungurahual. Estos datos fueron recogidos por las mismas comunidades a través del empleo de matrices de monitoreo comunitario.

Entre el 2004 y el 2007 el IIAP ejecutó otro proyecto en la parte baja de la misma cuenca, incluyendo la Reserva Nacional Allpahuayo-Mishana, esta vez financiado por el Gobierno de Finlandia con fondos de canje de deuda por naturaleza: Proyecto Diversidad Biológica de la Amazonía Peruana Perú - Finlandia (BIODAMAZ Fase II; Álvarez et al. 2004). Las líneas maestras de trabajo con comunidades locales replicaron y mejoraron las líneas estratégicas aplicadas en el Proyecto Nanay, especialmente el enfoque de co-gestión, dado que este proyecto se ejecutó en el escenario de un área protegida, donde las comunidades comparten las competencias de gestión de recursos naturales con los técnicos y representantes del Estado. Durante esta experiencia también se logró grandes avances en la aplicación del modelo de manejo comunal de recursos de la biodiversidad, con resultados notables en la disminución de las amenazas para la biodiversidad y la degradación de los ecosistemas. En las Figuras 1 y 2 se aprecia, por ejemplo, la significativa disminución del ingreso de extractores ilegales de los recursos más importantes para la economía local (hojas de la palmera irapay y de la madera redonda de varillales) en la Reserva Nacional Allpahuayo-Mishana (también esta información es fruto de la aplicación de matrices de monitoreo comunitario).

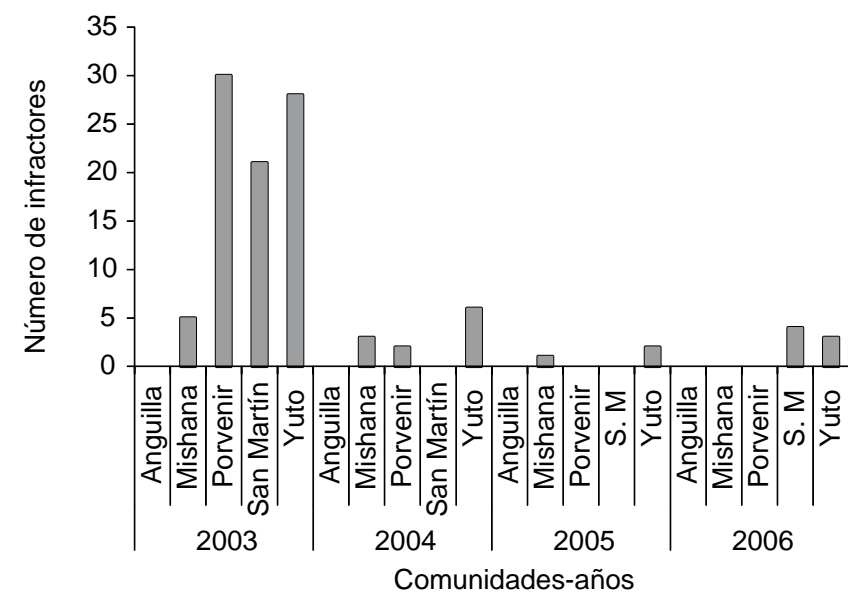

Figura 2. Índice de infractores de madera redonda en comunidades de la la Reserva Nacional Allpahuayo-Mishana (RNAM) (Fuente: Informes finales del Proyecto BIODAMAZ Fase II. IIAP, Iquitos, 2007. http://www.iiap.org.pe/biodamaz/faseii/avances/Doc_tec.htm)

En esta reserva se produjo también una substancial disminución de las prácticas de cosecha destructiva entre los pobladores, otra de las amenazas más importantes para la comunidad y para la biodiversidad. Como muestra presentamos el caso de la palmera irapay y madera redonda, los dos recursos más importantes en términos económicos para las comunidades de la zona (Figs. 3 y 4; Álvarez et al. 2004).

Como resultado de la aplicación de medidas de manejo adaptativo por las comunidades locales se produjo una substancial

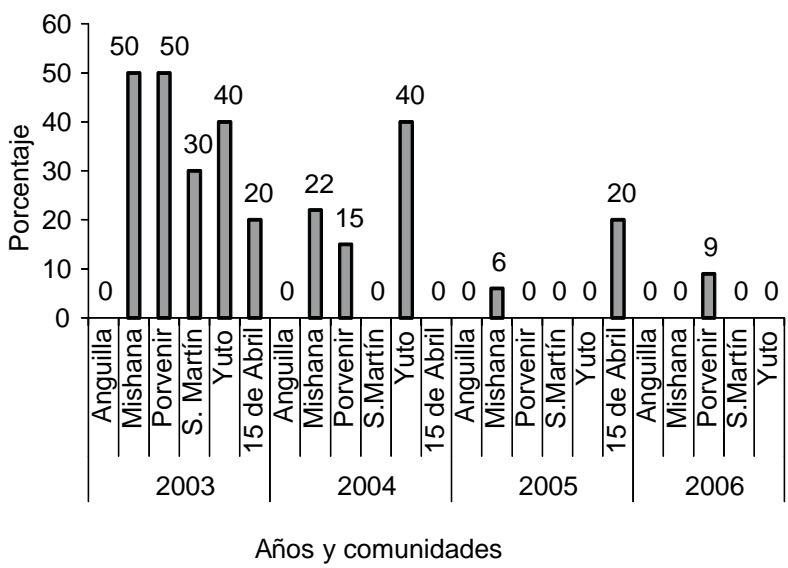

Figura 3. Porcentaje de familias que efectúan prácticas destructivas de cosecha de irapay. 


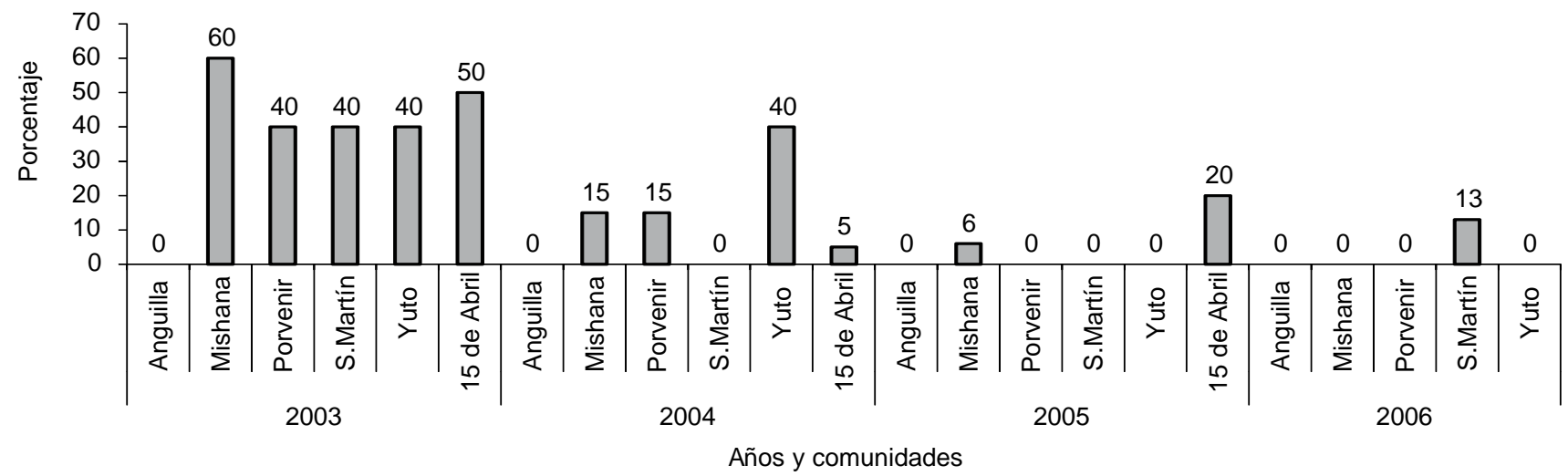

Figura 4. Porcentaje de comuneros que efectúan malas prácticas para el recurso madera redonda (Fuente: Informes finales del Proyecto BIODAMAZ Fase II. IIAP, Iquitos, 2007. http://www.iiap.org.pe/biodamaz/faseii/avances/Doc_tec.htm)

recuperación del recurso pesquero en los cuerpos de agua de la Reserva Nacional Allpahuayo - Mishana - RNAM, tal como se puede comprobar en la Figura 5, que mide el promedio de peces capturados por faena con distintas técnicas de pesca (captura por unidad de esfuerzo). Las medidas de manejo, propuestas por las mismas comunidades y aprobadas en acta de asamblea comunal incluyeron, entre otros aspectos: prohibición de pesca comercial por foráneos, control de métodos destructivos de pesca (tóxicos y explosivos, redes no selectivas, honderas y arrastradoras), regulación del tamaño de la malla de redes-trampa, límites en el volumen de capturas por pescador con fines comerciales, prohibición de pesca comercial durante la época de desove, y control de tala de bosques ribereños (Álvarez et al. 2004).

Cabe destacar que solo se monitoreó directamente las capturas pesqueras porque este recurso se recupera de forma relativamente rápida, a diferencia de la fauna silvestre terrestre y de la flora, y es factible monitorear las capturas por unidad de esfuerzo trabajando con los pescadores locales. En relación con otros recursos, se optó por monitorear solo las intervenciones de la población sobre el recurso, y no directamente el recurso, dado que: i) el monitoreo directo es sumamente caro y de mediano/largo plazo, y fuera del alcance por tanto del horizonte de un típico proyecto de cooperación al desarrollo y la conservación; y ii) los cambios en la composición y abundancia de los recursos son difícilmente distinguibles en el corto plazo de las fluctuaciones poblacionales naturales (de la fauna silvestre en especial) o del 'ruido estadístico', y por tanto son irrelevantes para los objetivos de un proyecto. El enfoque del monitoreo ha sido netamente participativo: las matrices de monitoreo fueron completadas con participación directa de

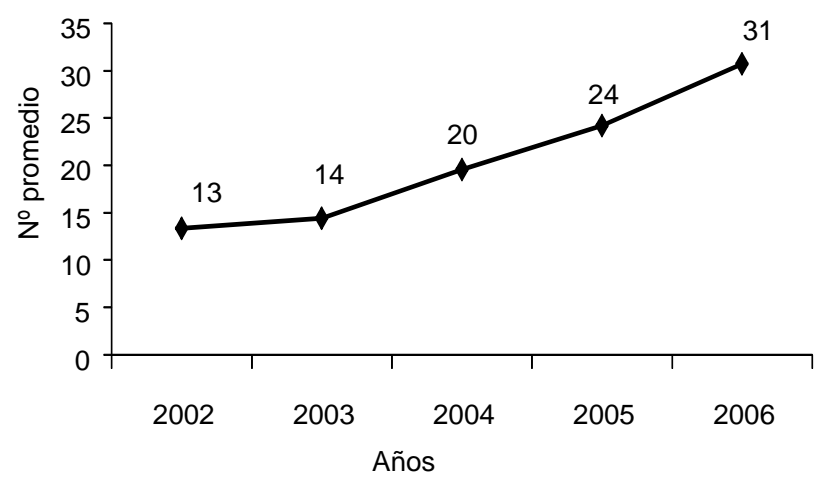

Figura 5. Evolución del número promedio de ejemplares pescados en la RNAM empleando diferentes técnicas de pesca (Fuente: Informes finales del Proyecto BIODAMAZ Fase II. IIAP, Iquitos, 2007. http:// www.iiap.org.pe/biodamaz/faseii/avances/Doc_tec.htm) las mismas comunidades, unas veces en asamblea comunal, otras en reuniones con líderes destacados de la comunidad. Si bien la información reflejada en las matrices de monitoreo comunitario -y en las figuras aquí presentadas- es "de grano grueso", porque se basa en datos referidos por la población, es muy útil y relevante para detectar tendencias en los patrones de uso de recursos de las comunidades, y para monitorear la disminución de las amenazas. No nos cabe ninguna duda de que son un indicador fiel del estado de conservación real de los recursos de flora y fauna silvestre.

Finalmente, entre el 2007 y el 2010 se ha estado ejecutando un proyecto de apoyo a la creación y gestión de áreas de conservación regional en la Región Loreto, con financiamiento de Fundación Moore (Proyecto de Apoyo al PROCREL, Programa de Conservación, Gestión y Uso Sostenible de la Biodiversidad en la Región Loreto). El Proyecto fue co-ejecutado por el IIAP, el Gobierno Regional de Loreto, y la organización no gubernamental Nature and Culture International - NCI. La primera área de conservación regional en ser creada y gestionada fue el Área de Conservación Regional Comunal Tamshiyacu-Tahuayo, en el año 2007, aunque se trabajó con las comunidades desde varios años antes. $\mathrm{Al}$ año siguiente fueron creadas otras dos, el $\mathrm{ACR}$ Ampiyacu-Apayacu, y el ACR Alto Nanay-Pintuyacu-Chambira.

Los logros de la aplicación del modelo de co-gestión comunal han sido muy significativos, especialmente en el ACR TamshiyacuTahuayo, donde las comunidades ya llevaban años gestionando esta zona, aún antes de ser declarada oficialmente como área de conservación regional. Con apoyo de organizaciones como Wildlife Conservation Society las comunidades de la cuenca del río Tahuayo han estado aplicando por más de una década medidas de manejo de fauna silvestre con gran éxito (Bodmer et al. 1997; Bodmer \& Puertas 2000, Novaro et al. 2000), y el modelo ha sido reconocido como uno de los mejores en gestión de bosques tropicales a nivel mundial (Congreso Forestal Mundial 2009, Buenos Aires). En el Perú también han recibido diversos reconocimientos y premios. El Proyecto de Apoyo al PROCREL impulsó el manejo comunal de otros recursos además de la fauna silvestre, incluyendo pesquerías, palmera irapay (Lepidocaryum tenue), palmera chambira (Astrocaryum chambira), palmera aguaje (Mauritia flexuosa), camu camu (Myrciaria dubia), entre otros recursos importantes para la población.

Las comunidades no solo han conseguido recuperar la productividad de sus bosques y ecosistemas acuáticos, y han mejorado su calidad de vida gracias a la abundancia de fauna silvestre, pescado y otros recursos: también han mejorado subs- 


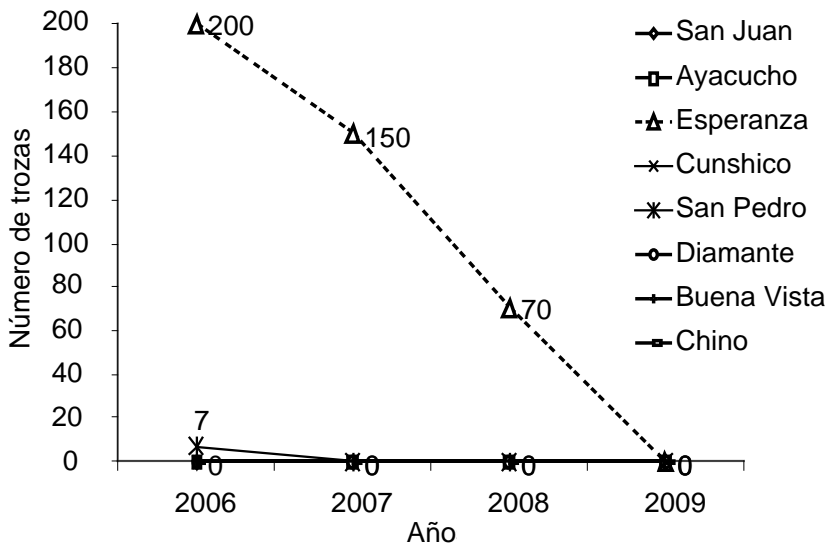

Figura 6. Número de infractores que ingresaron a extraer madera.

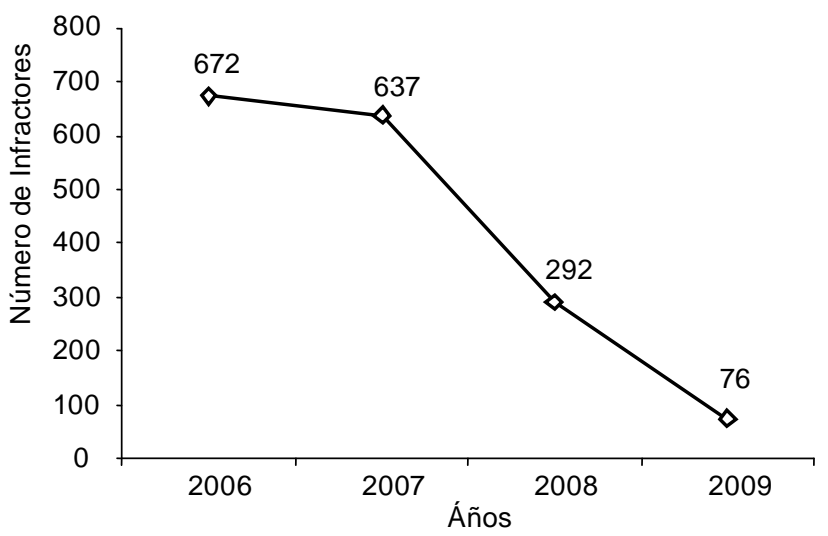

Figura 8. Descenso de ingreso de infractores al área de influencia del Área de Conservación Regional Comunal Tamshiyacu-Tahuayo (ACRACTT).

tancialmente su economía, gracias al impulso de actividades económicas de valor agregado, incluyendo la elaboración y exportación de artesanías y el ecoturismo. Más de cien familias han mejorado sus ingresos entre 100 y $500 \%$ en los últimos tres años gracias a la exportación a EE. UU. de artesanías de la fibra de la palmera chambira (PROCREL 2010)

Con base en la aplicación de matrices de monitoreo comunitario se ha podido determinar el dramático descenso de las amenazas para los recursos de la biodiversidad en las zonas donde se implementó el proyecto (Figs. 6 - 9, PROCREL 2010)

También se ha comprobado una notable recuperación de las pesquerías por efectos del manejo comunitario (del orden del $40 \%$ en cinco años), en especial en la Quebrada Yanayacu, área de amortiguamiento del Área de Conservación Regional Comunal Tamshiyacu-Tahuayo, en donde el trabajo con comunidades es más reciente y se ha monitoreado la captura por unidad de esfuerzo desde el inicio de la aplicación de las medidas de manejo (Fig. 10; PROCREL 2011)

\section{Conclusiones}

Las comunidades locales pueden no ser el 'modelo perfecto' de gestión del bosque amazónico. Están compuestas por personas, y las personas cometen errores y también pueden llegar a ser cortoplacistas y egoístas; los indígenas y ribereños amazónicos no son una excepción, no existe el "noble salvaje" (Alvard 1993). La llamada "tragedia de los bienes comunes" es actualmente una de las más graves amenazas para la salud de los ecosistemas amazónicos: la falta de control del Estado y el colapso de las

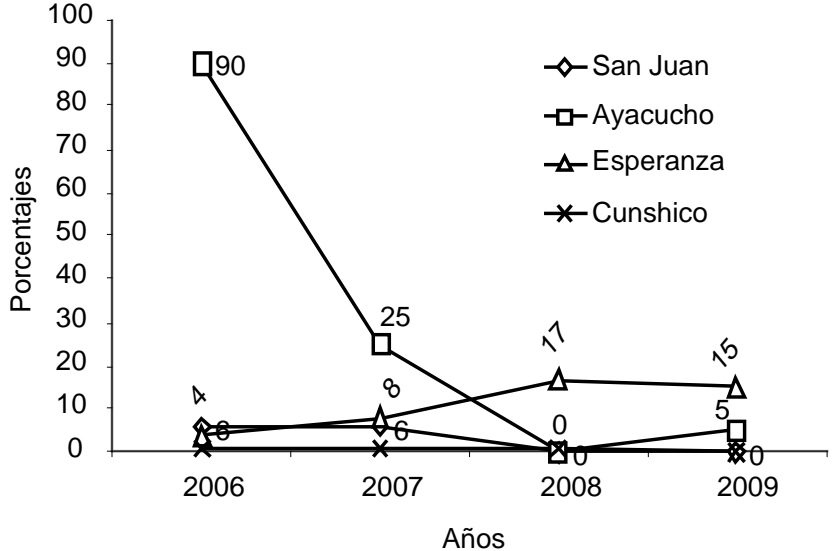

Figura 7. Porcentaje de comuneros que realizan practicas destructivas para pescar.

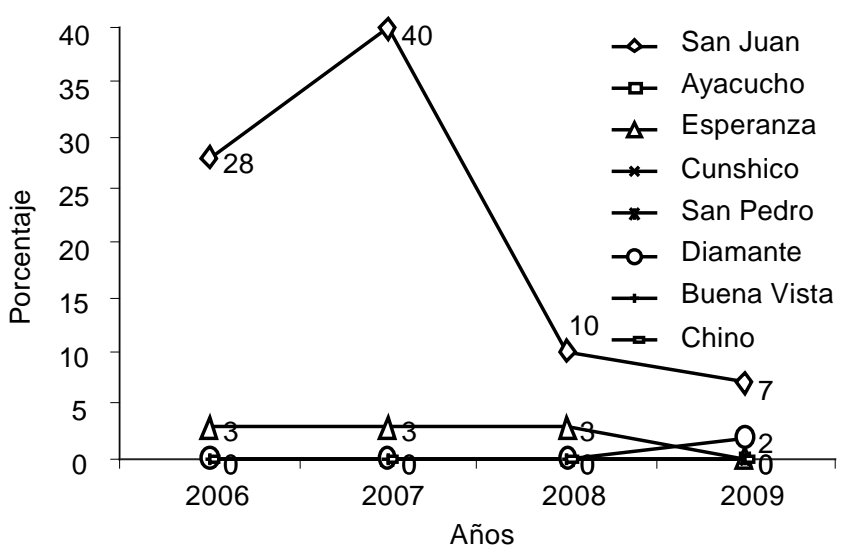

Figura 9. Porcentaje de comuneros que extraen palmeras para techado con prácticas destructivas.

sociedades indígenas favorecen la extracción indiscriminada y sin medidas de manejo de recursos de flora y fauna (Smith 2003; Ostrom 2003). Y la tragedia de los bienes comunes también puede ocurrir dentro de las comunidades, cuando no ejercen control sobre las acciones de sus miembros.

Los resultados de los proyectos impulsados por el IIAP y algunas otras organizaciones muestran que sí se puede mejorar de la calidad de vida y de la economía de las poblaciones amazónicas al mismo tiempo que se revierten los procesos de pérdida de bosques y degradación de hábitats, contribuyendo por tanto a conservar de forma efectiva y sostenible la diversidad biológica en

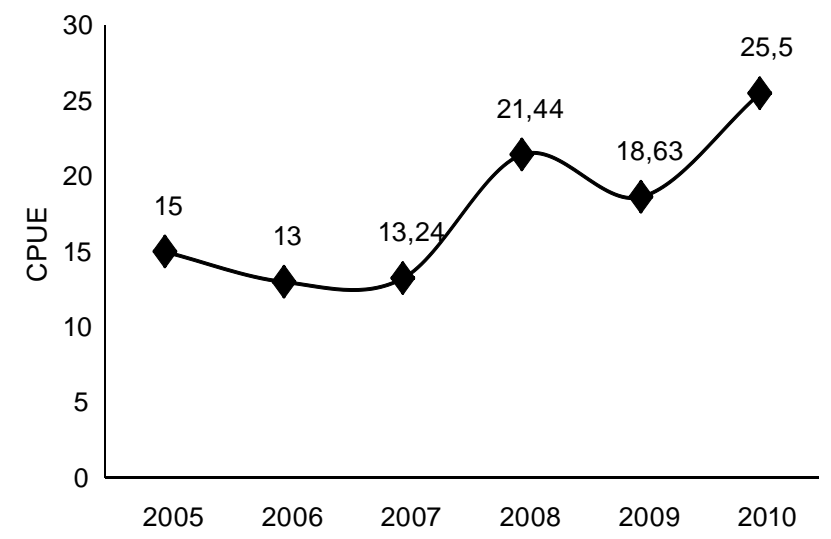

Figura 10. Captura de pescado por unidad de esfuerzo (número de individuos capturados/número promedio de red-trampa utilizadas) en la Quebrada Yanayacu, Área de Conservación Regional Comunal Tamshiyacu-Tahuayo (ACRACTT). 
grandes espacios naturales. No cabe duda de que las comunidades pueden ser mucho mejores gestoras que el Estado, al menos en el escenario de la Amazonía baja del Perú.

Sin embargo, es más que claro que actualmente las comunidades amazónicas necesitan una buena dosis de acompańamiento y capacitación para que salgan de la vorágine extractivista en la que están metidos desde hace dos o tres generaciones: extracción descontrolada de recursos de flora y fauna - empobrecimiento creciente de la población - más presión sobre los recursos. Es un círculo vicioso que se agrava ańo tras ańo, a medida que se articulan más con el mercado y requieren más bienes de consumo (Trujillo 2009). Para garantizar la sostenibilidad económica del modelo de "conservación efectiva" de grandes paisajes es indispensable el desarrollo de alternativas productivas con articulación al mercado.

Un aspecto importante a tener en cuenta en estas experiencias es que se ha obtenido avances significativos enfrentando dos de los graves retos en la Amazonía: impulsar el desarrollo sostenible de las comunidades locales y promover la conservación efectiva de los bosques y otros ecosistemas. Cerca de dos millones de hectáreas están ahora "efectivamente" protegidas en varias áreas protegidas de nivel nacional y regional en Loreto; en ellas se recuperan ecosistemas y recursos sobre explotados en el pasado, de fauna y flora, mientras que se aprovechan con medidas de manejo recursos de fauna y productos forestales diferentes a la madera para beneficio de las comunidades.

En las áreas con mayor tiempo de gestión las comunidades han mejorado tanto su calidad de vida (dada la recuperación de la fauna y la flora que constituyen la base de su economía, y gracias al involucramiento en actividades económicas de agregación de valor y comercialización de productos de la biodiversidad) que se han convertido en un modelo de desarrollo para otras áreas y para otras regiones. El Gobierno Regional de Loreto ha asumido al PROCREL y sus líneas estratégicas como un proyecto emblemático en el marco de su visión de "Región Productiva"; y varios pueblos indígenas han mostrado interés en replicar el modelo de co-gestión comunal y conservación productiva en sus zonas, mientras que en la Región Ucayali se está ejecutando un proyecto similar al de Loreto. Las regiones Madre de Dios y Amazonas, por su parte, han mostrado también interés en replicar el modelo.

\section{Literatura citada}

Alvard M. S. 1993. Testing the "Ecologically Noble Savage" Hypothesis: Interspectific prey choice by Piro Hunters of Amazonian Peru. Human Ecology 21(4): 355 - 387.

Alvarez J. 2006. Gestión comunal y territorio: lecciones aprendidas de la cuenca del Nanay (Amazonía norperuana) para el manejo de la fauna silvestre amazónica. Revista electrónica Manejo de fauna silvestre en la Amazonía. No 1, Vo. 1.

Álvarez J. 2007. Comunidades locales, conservación de la avifauna y de la biodiversidad en la Amazonía peruana. Rev. Peru. Biol. 14(1): 151-158.

Álvarez J., F. Rojas M. y A. J. Araujo. 2004. Una experiencia de manejo comunal de los recursos del bosque en la Reserva Nacional Allpahuayo - Mishana. Documento Técnico $\mathrm{N}^{\circ} 11$, BIODAMAZ, IIAP. http://www.iiap.org.pe/ biodamaz/faseii/download/literatura_gris/Articulos $\% 20$ cient $\% \mathrm{C} 3 \%$ ADficos/ [Acces 03.07.2011].

Anderson. A. (Ed.) 1989. Alternatives to Deforestation Steps Toward Sustainable Uses of Amazonian Forests. Columbia University Press, New York.
Asner G.P., G.V.N. Powell, J. Mascaro, et al. 2003. Who should pay for tropical conservation and how could the costs be met? Oryx 37: $238-250$.

Balmford A. \& T. Whitten 2003. Who should pay for tropical conservation and how could the costs be met? Oryx 37: 238-250.

Bodmer R. 2000. Integrating hunting and protected areas in the Amazon. Pp. 277-290 en N. Dunstone and A. Entwistle (Eds.) Future Priorties for the Conservation of Mammals: Has the Panda had its Day? Cambridge University Press, Cambridge, UK.

Bodmer R. \& P. Puertas 2000. Community-based comanagement of wildlife in the Peruvian Amazon. Pp. 395-409 en J. Robinson and E. Bennett (Eds.) Hunting for Sustainability in Tropical Forests. Columbia University Press, New York.

Bodmer R.E., J.W. Penn, P.E. Puertas, L. Moya I. \& T.G. Fang. 1997. Linking conservation and local people through sustainable use of natural resources: Community-based management in the Peruvian Amazon. Pp. 315-358 en C. Freese (Ed.) Harvesting Wild Species. John Hopkins University Press.

Brown K. 2003. Three challenges for a real people-centred conservation. Global Ecology and Biogeography, 12, 89-92.

Bruner A.G., R.E. Gullison, R.E Rice \& G.A.B. da Foncesca. 2001. Effectiveness of parks in protecting tropical biodiversity. Science 291: 125-128.

Butler R.A. \& W.F. Laurance. 2009. Is oil palm the next emerging threat to the Amazon? Tropical Conservation Science Vol. 2(1):1-10.

CADMA. 1992. Amazonía sin mitos. Comisión Andina de Desarrollo y Medio Ambiente. Ed. Banco Interamericano de Desarrollo/PNUD/TCA.

Campbell L.M. \& A. Vainio-Mattilia. 2003. Participatory development and community based conservation: opportunities missed for lessons learned. Human Ecology, 31, 417-437.

CIAM. 2011. La Amazonía vale un Perú. Consejo Interregional Amazónico - CIAM, documento técnico. Lima.

Clad J. 1984. Conservation and indigenous peoples: a study of convergent interests. Cultural Survival Quarterly 8: 68-73.

Clay J. 1988. Indigenous Peoples and Tropical Forests. Models of Land Use and Management from Latin America. Cultural Survival. Inc. Cambridge, MA. Cultural Survival Report 27: 69-73.

CSD - Commission on Sustainable Development (2004) Partnerships for Sustainable Development: Report of the Secretary General. Http://www.un.org/esa/sustdev/csd/csd12/ csd12_docs.htm [Acces. 23.12.2010].

Cooke B. \& U. Kothari (Eds.) 2001. Participation: The New Tyranny? Zed Books, London, UK, 207 pp.

Danielsen F., M. M. Mendoza, A. Tagtag, et al. 2007. Increasing Conservation Management Action by Involving Local People in Natural Resource Monitoring. Ambio 36, 7: 566-570.Davis S. H. and A. Wali. 1994. Indigenous Land Tenure and Tropical Forest Management in Latin America. Ambio Vol. 23 No. 8.

Davis S. H. \& A. Wali. 1994. Indigenous Land Tenure and Tropical Forest Management in Latin America. Ambio Vol. 23 No. 8.

De Soto H. 2010. La Amazonía no es Avatar. El Comercio, Perú 05.06.2010.

Ferraro P. J. \& S. K Pattanayak. 2006. Money for nothing? A call for empirical evaluation of biodiversity conservation investments. PLoS Biology, 4, e105.

García Villacorta R. 2010. Identificación de Procesos Ecológicos y Evolutivos Esenciales para la Conservación de la Biodiversidad en la Región Loreto. Documento Técnico Proyecto Apoyo al Programa de Conservación, Gestión y Uso Sostenible de la Biodiversidad en la Región Loreto. $122 \mathrm{pp}$. Iquitos. 
Gasché J. 1999. Desarrollo rural y pueblos indígenas amazónicos. Ed. Abya-Yala. Quito.

Gasché J. 2004: Una concepción alternativa y crítica para proyectos de desarrollo en la Amazonía. Pp. 105-118 en J. Gasché (Ed.): Crítica de proyectos y proyectos críticos de desarrollo. Iquitos, Instituto de Investigaciones de la Amazonía Peruana.

Gasché J. 2010. Conferencia en la Universidad Nacional de Colombia. IIAP, manuscrito, $25 \mathrm{pp}$

Getz W.M., Fortmann, L., Cumming, D., et al. (Eds.) 1997. Social Change and Conservation: Environmental Politics and Impacts of National Parks and Protected Areas. Earthscan, London, UK.

Ghimire, K.B. \& M.P. Pimbert (Eds.) 1997. Social Change and Conservation: Environmental Politics and Impacts of National Parks and Protected Areas. Earthscan, London, UK.

Goodman D. \& A. Hall. A. (Eds.) 1990. The Future of Amazonia: Destruction or Sustainable Development. St. Martin's Press, New York.

Guha R. 1997. The authoritarian biologists and the arrogance of anti-humanism: wildlife conservation in the third world. The Ecologist 27: 14-20.

Holt F. L. 2005. The catch-22 of conservation: indigenous peoples, biologists and cultural change. Human Ecology 33: 199-215.

Houtart F. 2011. El escándalo de los agrocombustibles en los países del Sur http://alainet.org/active/47497. [acces. 10.06.11].

IIAP. 2004. Informe final del Proyecto "Conservación de la biodiversidad y manejo comunal de los recursos naturales en la cuenca del río Nanay" Banco Mundial/GEF. Documento Técnico, Instituto de Investigaciones de la Amazonía Peruana, Iquitos.

Johnson A. 1989. How the Machiguenga manage resources: conservation or exploitation of nature? Advances in Economic Botany 7: 213-222.

Novaro A., Redford, K. \& R. Bodmer. 2000. Effect of hunting in source-sink systems in the Neotropics. Conservation Biology 14(3): 713-721.

Ostrom E. 2003. Reformulando los bienes comunes. Pp. 48-77 en Smith R. Ch. \& D. Pinedo (Eds.). El cuidado de los bienes comunes. Gobierno y manejo de los lagos y los bosques en la Amazonía. Lima, Ed. IEP.

Parry L., C. A. Peres, B. Day \& S. Amaral. 2010. Rural-urban migration brings conservation threats and opportunities to Amazonian watersheds. Conservation Letters xx 1-9.

Peres C. 1994. Indigenous reserves and nature conservation in Amazonian forests. Conservation Biology 8(2): 586-588.

Peres C. 2000. Evaluating the impact and sustainability of subsistence hunting at multiple Amazonian forest sites. Pp. 31-56 en J. Robinson and E. Bennett (Eds.) Hunting for Sustainability in Tropical Forests. Columbia University Press, New York

Phillips O., G. López-González, Y. Malhi, et al. 2009. Drought Sensitivity of the Amazon Rainforest. Science 323: 1344-1347.

Porter-Bolland L., E. A. Ellis, M. R. Guariguata, et al. 2011. Community managed forests and forest protected areas: An assessment of their conservation effectiveness across the tropics. Forest Ecol. Manage. doi:10.1016/j.foreco.2011.05.034

Posey D. \& B. Balee (Eds.) 1989. Resource Management in Amazonia: Indigenous and Folk Strategies. New York Botanical Garden. New York. Advances in Economic Botany. Vol. 7.

PROCREL 2011. Informe de monitoreo participativo de la pesca en la Quebrada Yanayacu, zona de amortiguamiento del Área de Conservación Regional Comunal Tamshiyacu-Tahuayo. Informe Técnico Programa de Conservación, Gestión y Uso Sostenible de la Biodiversidad en la Región Loreto - PROCREL. Iquitos.
PROCREL. 2010. Informe de monitoreo participativo en la zona de amortiguamiento del Área de Conservación Regional Comunal Tamshiyacu-Tahuayo. Informe Técnico Programa de Conservación, Gestión y Uso Sostenible de la Biodiversidad en la Región Loreto - PROCREL. Iquitos.

Pyhälä Aili. 2003. Productive Conservation in Amazonia: Institutions, Participation and Markets. PhD Thesis submitted to the School of Development Studies, University of East Anglia, U.K.

Redford KH. \& A.M. Stearman. 1993. Forest-dwelling native Amazonians and the conservation of biodiversity: interests in common or in collision? Conservation Biology 2: 248-255.

Redford K.H. 1992. The empty forest. Bioscience 42: 412-422.

Redford K.H. \& S. Taber. 2000. Writing the wrongs: developing a safe-fail culture in conservation. Conservation Biology 14: $1567-1568$.

Roe D. \& M. Jack. 2001. Stories from Eden: Case Studies of Community-based Wildlife Management. Evaluating Eden Series 9. International Institute for Environment and Development, London, UK.

Sanderson S. E. \& K. H. Redford. 2003. Contested relationships between biodiversity conservation and poverty alleviation. Oryx 37: 389-390.

Santoyo A. 2004. Representaciones y programa de cambio en la Amazonía durante el siglo XIX: Análisis del pensamiento civilizador colombiano. Pp. 13-28 en J. Gasché (Ed.) Crítica de proyectos y proyectos críticos de desarrollo. Iquitos, Instituto de Investigaciones de la Amazonía Peruana.

Schwartzman S. \& B. Zimmerman. 2005. Conservation alliances with indigenous peoples of the Amazon. Conservation Biology 19: 721-727.

Sheil D. \& A. Lawrence. 2004. Tropical biologists, local people and conservation: new opportunities for collaboration. Trends Ecol. Evol. 19, 634-638.

Smith D Ch. 2003. Los bienes comunes y su gestión comunitaria: conceptos y prácticas. Pp. 13-30 en D. Ch. Smith \& D. Pinedo (Eds.). El cuidado de los bienes comunes. Gobierno y manejo de los lagos y los bosques en la Amazonía. Lima, Ed. IEP.

Smolker R., B. Tokar., A. Petermann, E. Hernández. 2007. El verdadero costo de los agrocombustibles: alimentación, bosques y clima. Global Forest Coalition, $80 \mathrm{pp}$.

Terborgh J. 1999. Requiem for Nature. Island Press, Washington, DC, USA.

Terborgh J., G. Nuñez-Iturri, N. C. A. Pitman, et al. 2008. Tree recruitment in an empty forest. Ecology, 89(6): 1757-1768

Trujillo Osorio C. 2009. Efectos de la integración al mercado sobre las formas de uso del bosque tropical en territorios indígenas del Amazonas. Tesis para acceder a la Maestría en Estudios Amazónicos de la Univ. Nacional de Amazonia (Leticia, Colombia).

Vandermeer J. \& I. Perfecto. 2007a. Tropical conservation and grass roots social movements: Ecological theory and social justice. Bulletin of the Ecological Society of America 88:171-175

Vandermeer J. \& I. Perfecto. 2007b. The agricultural matrix and a future paradigm for conservation. Conservation Biology 21:274-277.

Vermeulen S. \& D. Sheil. 2007. Partnerships for tropical conservation. Oryx 41(4): 434-440.

Wright S. J. \& H.C. Muller-Landau. 2006. The Future of Tropical Forest Species. BIOTROPICA 38(3): 287-301.

Wright A. \& W. Wolford. 2003. To inherit the earth: the landless movement and the struggle for a new Brazil. Food First Books, Institute for Food and Development Policy, San Francisco, California, USA. 